\title{
Some Thoughts Regarding the Narrative of the Current Nosological Schema and their Ramifications for Major Mental Disorders with a Focus on Schizophrenia
}

\author{
Nicholas Pediaditakis \\ Department of Psychiatric Medicine, ECU Brody School of Medicine, Greenville, NC, USA \\ Email: nicholaspediaditakis@gmail.com
}

How to cite this paper: Pediaditakis, N. (2020) Some Thoughts Regarding the Narrative of the Current Nosological Schema and their Ramifications for Major Mental Disorders with a Focus on Schizophrenia. Open Journal of Psychiatry, 10, 64-68.

https://doi.org/10.4236/ojpsych.2020.102008

Received: February 4, 2020

Accepted: April 7, 2020

Published: April 10, 2020

Copyright ( 2020 by author(s) and Scientific Research Publishing Inc. This work is licensed under the Creative Commons Attribution International License (CC BY 4.0).

http://creativecommons.org/licenses/by/4.0/ (c) (i) Open Access

\begin{abstract}
The current nosological schema for major mental disorders does not capture all the clinical phenomena, especially those being observed longitudinally (i.e., six decades of clinical observations of over eight thousand patients suffering with emotional problems of which three thousands of them suffer from major mental disorders, spanning two to three generations by the author). The objective of this article is to point out that these overlooked in their collective significance phenomena-even though they are noted by the clinicians, are not part of the categorical guidelines of the current schema. They have ramifications for better conceptualizing the road-guide in our future research for these baffling, major mental disorders, including schizophrenia, bipolar affective disorder, obsessive-compulsive disorder (OCD), anxiety, and attention deficit hyperactivity disorder (ADHD), which are sharing a common, neuro-developmental origin.
\end{abstract}

\section{Keywords}

Major Mental Disorders, Schizophrenia, Neurology, Psychopathology

\section{Introduction}

There exists a need for a better narrative for the current categorical guidelines of the major mental disorders (i.e., schizophrenia, anxieties, bipolar affective and obsessive-compulsive disorders). Considering such a narrative encompassing clinical phenomena shared by all the major mental disorders will be a more fruitful guide for delineating the mechanisms of their psychopathology. This 
outline is a review on the topic based at length in a previously, published article titled "An Emerging Natural History in the Development, Mechanisms and Worldwide Prevalence of Major Mental Disorders” [1].

\section{Subjects and Method}

The clinical phenomena of schizophrenia shared by the rest of the other major mental disorders apparent in longitudinal observations at present are over the horizon of our scrutiny. They include the following; first, the longitudinal observations of the same individual patients show frequent shifts from one condition to another. Thus, bipolar affective disorder shifts to obsessive-compulsive disorder, and schizophrenia to severe anxiety. Diagnosing these phenomena as co-morbidities mask the fact that it is the same disease with its frequent shift in its syndrome. Second, the group gene loci associated with schizophrenia and the other four conditions (i.e., attention-deficit/hyperactivity disorder (ADHD), bipolar affective disorder, obsessive-compulsive disorder (OCD), and incapacitating anxiety) [2] certainly code for the premorbid personalities of the mentioned major mental disorders and not the expression of the disorders themselves, since, the former invariably antedates the latter [3]. Focusing on schizophrenia, each of the variant syndromes of the disorder occurs probabilistically on some "at-risk" individuals whose pre-existing extreme, temperamental variance confers vulnerability for the expression of the disorder. Even the recently found group of genes directly responsible for schizophrenia are actually coding for extreme temperamental lopsidedness, thus, conferring vulnerability in individuals with such extreme temperament for developing the disorder.

The extreme premorbid personality (a.k.a., temperament, idiosyncrasy, physis, personality traits, character) is invariably present in schizophrenia as well in the remaining aforementioned disorders. Temperament in its normal form is made up of components/traits whose origins, according to E.O. Wilson [4], emanate from two evolutionary pressures: the ones that result on the individual, such as an "inner-directed," autonomous individual who feels comfortable in being alone with self-autonomy and with a paucity of social interactions (e.g., alienation), those above-mentioned traits, again normally, (albeit, not in the pronounced extreme form as in the case of major mental disorders especially schizophrenia), they coexist side-by-side with those traits emanating from evolutionary pressures on the social experience of humans, such as empathy, altruism, cooperativeness, eagerness for social interactions, and group identity. Together, they make up the human nature but, in an unamalgamated mix. They result in an uneasy, conflicting existence with oneself and others [5]. All these traits again normally emanating from the two evolutionary pressures mentioned above are occurring in a different recipe allocation of their traits for each individual, thus making up the infinite variability of temperamental traits. Evolutionarily, they confer flexibility and robustness for our human tribe. In the case of schizophrenias, on account of the extreme lopsided temperament emanating 
mainly from the evolutionary pressures on the individual, the variable symptoms that define the disorder should be considered as a clinical expression of a phase shift, i.e., a switch to the pathologically, ordered phase of the overall operating mode of brain function. This mode is based on an emergent quality of complexity, which normally ensures the synchrony, coordination, subtlety, and flexibility in the expression of the components of the various higher faculties of the brain when this shift effects mood modulation. It results in the bipolar disorders, when it effects, the thought processes, and alignment of thoughts with feelings, an appropriateness of behavior with the demands made by the environment. It results in schizophrenia when it effects the algorithmic faculty (i.e., scheduling and fore-planning) results in obsessive-compulsive disorder, and when it effects the appropriate responses to the external world results in odd behavior.

The initial expression of the periodic epiphenomena, comprising the major mental disorders-superimposed on the pre-existing "lopsided," temperament, (i.e., premorbid trait) should be considered-at least in the beginning-similar to atrial fibrillation. Both atrial fibrillation and the initial clinical phenomenon of schizophrenia signifying the phase shift of coordination and synchrony and both recover by application of electroshock, at least temporarily. Fifth, these symptoms are made of antithetical substitutes of an either/or nature; thus, apathy alternates with explosive outburst, and the appearance of ambivalence (i.e., rapid shifting attitude and feelings for the same person or object) and frantic activity being replaced by lassitude. These phenomena are not captured by the terms used by the current schema of negative symptoms. Specifically, the term negative symptoms which are an awkward neologism that obscures rather than elucidate the phenomena at hand, preventing us to notice their collective significance as being the result of a phase shift of the overall normal mode of synchrony.

Frequently, schizophrenia's phenomena overlap with those of the rest of the previously mentioned, four, major mental disorders, while often even alternating with each other [6] [7].

In a parallax view of the current nosological schema, the symptoms of schizophrenia can be divided into three parts; the traditional Bleuler's four A's, i.e., disordered thinking (association), emotional flatness (apathy), alternating with explosive bouts, and the presence of autism in its broad, Greek definition of "preoccupation with oneself" and finally, ambivalence (the coexistence of contradictory sentiments or attitudes towards a subject) and additionally, inappropriate behavior to the environment. Often, pre-existing aloofness/apathy is felt by the individual himself, as an inner void conducive to a malignant boredom, a "dread beyond telling", and disconnectedness from their fellow humans, even though it does not preclude having social skills facilitating interactions and masking their felt alienation. Nevertheless, patients will readily confirm such feelings when asked.

It's important to note that the occurrence of concomitant occurrence of psychotic phenomena are similar to the ones that occur in other assaults on brain function, such as, trauma, toxicity, or high fever with the sole exception the de- 
lusions are more in their contend "narcissistic." Finally, constituting the third part of the disorder is the underlying, extreme temperamental traits previously mentioned which remain the same in remissions. Thus, the presence of psychosis is currently erroneously assigned as pathognomonic significance.

Significantly, the $1 \%$ worldwide prevalence of schizophrenia-irrespective of culture, persists in spite of the evolutionary pressure for its extinction. Since the condition appears early, the patients die earlier, and they have low fertility [7] [8] [9]. Such persistence can be explained evolutionarily as a trade-off between the extreme lopsided temperament that confers vulnerability to schizophrenia, on the one hand, and the advantageous creative ability conferred by this very extreme temperament, on the other [10]. The Aristotelian adage that "there is no genius without a touch of madness" is correct. Indeed, the presence of an extreme temperamental type in patients acts as an enabling factor in expressing creativity. It liberates these individuals from the constraints of social algorithms. Now, the individual is able to think in alternatives, to discern novel patterns in science, to create emotionally stirring pieces of art and music. Others of this type even become notable social leaders and politicians who recruit obedience and loyalty of the masses [11]. Not all geniuses become schizophrenic, and not all patients who have a "lopsided" temperament become geniuses. For that, they need intellect, curiosity, and persistence of effort. It is important to note that the above points are applied similarly to $\mathrm{OCD}$, bipolar affective disorder, and severe anxieties [12].

\section{Results and Conclusions}

In taking into consideration the postulates presented above can act as facilitating guides in research for delineating the baffling major mental disorders [13].

To recapitulate: first, the clinical manifestations of schizophrenia constitute periodic epiphenomena on a pre-existing, extreme temperamental variance at least in the beginning. Second, in addition, such clinical manifestations are the results of the phase shift of the overall operating mode of brain function-again overlooked in its significance which normally confers elegance, subtlety, ability to accommodate ambiguity shifting to a mode of an either/or pathological expression of symptoms made up of antithetical substitutes such as ambivalence, apathy alternating with explosive emotional outbursts, and gross inappropriate responses to environmental demands. They are readily recovered to the normal mode temporarily with the application of electroshock. The third manifestation made up of the psychotic phenomenon is comprised of delusions, and hallucinations which are similar to those expressed in any assault on brain function, such as brain trauma, high fever, and toxicity. And yet, currently the psychosis is assigned erroneously pathomemnonic significance.

\section{Conflicts of Interest}

The author declares no conflicts of interest regarding the publication of this paper. 


\section{References}

[1] Pediaditakis, N. (2016) An Emerging Natural History in the Development, Mechanisms and Worldwide Prevalence of Major Mental Disorders. Open Neurology Journal, 10, 149-154. https://doi.org/10.2174/1874205X01610010149

[2] Lee, S.H., Ripke, S., Neale, B.M., et al. (2013) Identification of Risk Loci with Shared Effects on Five Major Psychiatric Disorders: A Genome-Wide Analysis. Lancet, 38, 1371-1379. https://doi.org/10.1016/S0140-6736(12)62129-1

[3] Pediaditakis, N. (2012) Origins and Mechanisms in the Development of Major Mental Disorders: A Clinical Approach. Journal of Behavioral Brain Science, 2, 269-275. https://doi.org/10.4236/jbbs.2012.22030

[4] Wilson, E.O. (2012) The Social Conquest of Earth. Liveright Pub Corp., NY.

[5] Nowak, M.A., Wilson, E.O. and Tarnita, N.A. (2010) The Evolution of Eusociality. Nature, 466, 1057-1062. https://doi.org/10.1038/nature09205

[6] Freeman, M.P., Freeman, S.A. and McElroy, S.L. (2002) The Comorbidity of Bipolar and Anxiety Disorders: Prevalence, Psychobiology and Treatment Issues. Journal of Affective Disorders, 68, 1-23. https://doi.org/10.1016/S0165-0327(00)00299-8

[7] Leboyer, M., Henry, C., Paillere-Martinot, M.L. and Bellivier, F. (2005) Age at Onset in Bipolar Affective Disorders: A Review. Bipolar Disorder, 7, 111-118. https://doi.org/10.1111/j.1399-5618.2005.00181.x

[8] Collingwood, J. (2018) Premature Death Rates Rising in Schizophrenia, Bipolar. Psychcentral. https://psychcentral.com/lib/premature-death-rates-rising-in-schizophrenia-bipolar -patients

[9] Giudice, M.D. (2010) Reduced Fertility in Patients' Families Is Consistent with the Sexual Selection Model of Schizophrenia and Schizotypy. Public Library of Science (PLoS ONE), 5, e16040. https://doi.org/10.1371/journal.pone.0016040

[10] Andreasen, N.C. (1987) Creativity and Mental Illness Prevalence Rates in Writers and their First-Degree Relatives. American Journal of Psychiatry, 144, 1288-1292. https://doi.org/10.1176/ajp.144.10.1288

[11] Pediaditakis, N. (2014) The Association Between Mental Disorders and Geniuses. Psychiatric Times, 31, No. 9.

[12] Pediaditakis, N. (1998) Shared Characteristics in the Clinical Expression and Pharmacological Responses of Mental Disorders and their Possible Collective Significance. Medical Hypotheses, 50, 347-352. https://doi.org/10.1016/S0306-9877(98)90124-4

[13] Pediaditakis, N. (2016) Revisiting the Major Mental Disorders and Updating the Nosological Schema: A Synthesis. Journal of Behavioral and Brain Science, 6, 93-98. https://doi.org/10.4236/jbbs.2016.62010 